\title{
Acute Handling Stress Modulates Methylphenidate-induced Catecholamine Overflow in the Medial Prefrontal Cortex
}

\author{
Douglas A. Marsteller, B.S., Madina R. Gerasimov, D.D.S., Wynne K. Schiffer, B.S., \\ Justin M. Geiger, B.S., Channing R. Barnett, B.S., Jana Schaich Borg, B.S., \\ Sabria Scott, B.S., Jill Ceccarelli, B.S., Nora D. Volkow, M.D., Patricia E. Molina, Ph.D., \\ David L. Alexoff, B.S.E., and Stephen L. Dewey, Ph.D.
}

\begin{abstract}
Although stress is an extensively investigated phenomenon, the effects of specific stressors on the pharmacologic activity of routinely administered drugs are less well characterized. We designed the present study to investigate the effect of handling stress on catecholaminergic responsivity following an acute methylphenidate (MP, Ritalin) challenge in the medial prefrontal cortex (mPFC). Norepinephrine (NE) and dopamine (DA) levels were simultaneously measured in 15-min samples of PFC dialysate using HPLC coupled with electrochemical detection. Sprague-Dawley rats were handled for $15 \mathrm{~min}$,
\end{abstract}

which produced an increase from basal extracellular DA and NE levels. Handling stress attenuates the DA response when administered $2 \mathrm{~h}$ prior to IP MP, whereas handling stress enhances the DA response when administered simultaneously with IG MP. These findings suggest that persistent alterations in mesocorticolimbic DA-ergic activity are induced by a short exposure to restraint stress as evidenced by the altered response to MP challenge.

[Neuropsychopharmacology 27:163-170, 2002] (C) 2002 American College of Neuropsychopharmacology. Published by Elsevier Science Inc.
KEY WORDS: Handling stress; Methylphenidate; Medial prefrontal cortex; Norepinephrine; Dopamine; Microdialysis

Although stress is an extensively investigated phenomenon, the effects of specific stressors on the pharmaco-

From the Chemistry Department (DAM, MRG, WKS, JMG, CRB, JSB, SS, JC, NDV, DLA, SLD), and Medical Department (NDV), Brookhaven National Laboratory, Upton, NY 11973, Department of Neurobiology \& Behavior (DAM, WKS, SLD), and Department of Psychiatry (NDV), State University of New York, Stony Brook, New York, 11794, and Department of Physiology (PEM), Louisiana State University, New Orleans, LA 70112.

Address correspondence to: Madina R. Gerasimov, DDS Chemistry Department, Brookhaven National Laboratory, Upton, New York, 11973, Tel.: (516) 344-4395; Fax: (516) 344-7902; E-mail: madina@bnl.gov

Received September 10, 2001; revised December 18, 2001; accepted January 8, 2002.

Online publication: $1 / 11 / 02$ at www.acnp.org/citations/ Npp011102223. logic activity of routinely administered drugs are less well characterized. In many cases, these specific stressors induce changes in biochemical and behavioral profiles. Dopamine (DA) and norepinephrine (NE) are two catecholamines shown to have increased release and utilization in the medial prefrontal cortex (mPFC) during stress (Feenstra et al. 1999; Nakane et al. 1994; Abercrombie et al. 1989; Shimizu et al. 1994). Numerous investigations have demonstrated preferential increases in mPFC DA overflow associated with stressful stimuli when compared with limbic regions (Abercrombie et al. 1989; Ihalainen et al. 1999; Feenstra et al. 1998). This augmented response might be due to cross innervation of the mPFC from several brain stem projections, such as the A10 pathway from the ventral tegmental area (VTA) and/or noradrenergic connections from the locus coeruleus (LC). More specifically, systemic stressors such as handling, restraint, or immobilization increase 
mPFC levels of DA by 150-300\% (Enrico et al. 1998; Feenstra et al. 1995), and NE levels by $160-250 \%$ (Nakane et al. 1994; Kawahara et al. 2000; Shimizu et al. 1994). These responses can be modulated by infusion of pharmacologic agents directly into the LC and the VTA, indicating the influence of these regions on $\mathrm{MPFC}$ function. Specifically, infusion of tetrodotoxin (TTX) into the LC results in a significant reduction of NE basal concentrations in the mPFC (Van Gaalen et al. 1997) and TTX infusion into the VTA reduces DA extracellular concentrations in the mPFC (Mathe et al. 1999).

We designed the present study to investigate the effect of handling stress on catecholaminergic responsivity following an acute methylphenidate (MP, Ritalin) challenge in the mPFC. Methylphenidate is a psychostimulant that binds with a similar affinity (Gatley et al. 1996) to the DA transporter (DAT) and the NE transporter (NET) which produces increases in extracellular brain concentrations of both neurotransmitters in rodents (Kuczenski and Segal 1997). We previously demonstrated that therapeutically relevant doses $(2-10 \mathrm{mg} / \mathrm{kg})$ of oral MP produce significant increases in the mesocorticolimbic DA of rodents (Gerasimov et al. 2000) and humans (Volkow et al. 2001). Using a low dose of MP $(5 \mathrm{mg} / \mathrm{kg})$, we elucidated the direct effect that handling stress has on MP-induced DA and NE overflow in the MPFC. MP $(10 \mathrm{mg} / \mathrm{kg})$ was administered intragastrically (IG), as this route avoids potential stress-induced responses associated with an intraperitoneal (IP) injection. To further explore the potential influence of handling stress on MP pharmacologic activity, we also administered MP via an IP injection two hours following a handling session. This response was then compared against the control group given saline injection under identical conditions. Finally, given the established relationship of MP-induced increases in DA to increased locomotor activity (Kuczenski and Segal 2001), we characterized the effects of handling stress on the locomotor response to MP.

\section{METHODS}

\section{Animals, Surgery and Dialysis}

Adult male Sprague-Dawley rats (250-350 g, Taconic Farms) were anesthetized with ketamine $\mathrm{HCl} /$ xylazine $(100 \mathrm{mg} / \mathrm{kg})$, and siliconized guide cannulae were stereotactically implanted in the PFC; $\mathrm{A}=+3.7 \mathrm{~mm}, \mathrm{~L}=$ $-1.0 \mathrm{~mm}, \mathrm{~V}=-1.0 \mathrm{~mm}$ (Paxinos and Watson 1986) one or two days prior to the microdialysis experiment. Animals randomly assigned to intragastric drug administration of drug had a polyethylene catheter placed in the stomach using aseptic surgical techniques. The catheter was exteriorized after anchoring with a staple in the nape of the neck. On the day of the microdialysis study, rats were placed in a bowl and microdialysis probes $(4.0 \mathrm{~mm}$, Bioanalytical Systems, BAS) were positioned within the guide cannulae at least $2 \mathrm{~h}$ prior to dialysate collection. Ringer's Solution (Henry Schein, Inc) was administered through the probe using a CMA/100 microinfusion pump (BAS) at a flow rate of $1.0 \mu \mathrm{l} / \mathrm{min}$. Dialysate samples were collected every fifteen minutes $(10 \mu \mathrm{l})$, injected online and analyzed until three sequential injections differed by less than $10 \%$. The average DA concentration of these three stable samples was defined as basal $(100 \%)$ and subsequent treatment values were transformed to a percentage of that baseline. At the end of each experiment, animals were euthanized with an overdose of IP chloral hydrate, according to the 1993 AVMA Panel on Euthanasia. All animals were used under an Institutional Animal Care and Use Committeeapproved protocol and with strict adherence to National Institutes of Health guidelines.

\section{Drug Treatment}

MP hydrochloride $(5 \mathrm{mg} / \mathrm{kg}$ ) (a racemic mixture of $d$-threoand l-threo-MP; Research Biochemicals International, Natick, MA) was dissolved in saline and injected IP. IG administration $(10 \mathrm{mg} / \mathrm{kg})$ was accomplished by dissolving MP in saline, then injection through the pre-implanted catheter, followed by a rinse with saline. These methods were used for microdialysis and locomotor activity studies.

\section{Handling Stress}

Animals were handled continuously for 15 min during stress. Handling sessions consisted of partial removal from the dialysis chamber, during which animals were continually restrained. Continuous restraint consisted of picking up the animal by front shoulders, hips, or tail, and changing positions of the animal in the hand throughout this time so that it was not allowed to escape.

\section{Protocols}

Nine different groups of animals were studied in the following manner: (1) handling alone; (2) intraperitoneal (IP) administration of MP (5 mg/kg); (3) handling followed two hours later by IP administration of MP, (5 $\mathrm{mg} / \mathrm{kg}$ ); (4) IG administration of MP (10 mg/kg); (5) IG administration of MP $(10 \mathrm{mg} / \mathrm{kg})$ followed by stress 15 min later. Identical protocols for groups 1, 2, 3, and 4 were used for locomotor studies.

\section{Biochemical Assay}

The HPLC system consists of a microbore column $(150 \times$ $1 \mathrm{~mm}, 5-\mu \mathrm{m}$ C18 column; BAS), a BAS LC-4C electrochemical transducer with a dual glassy carbon electrode set at $+650 \mathrm{mV}$, and a double pen chart recorder. The mobile phase (flow rate $=1.0 \mathrm{ml} / \mathrm{min}$ ) consists of $1 \mathrm{~L}(2 \mathrm{~g}$ 
$\mathrm{NaH}_{2} \mathrm{PO}_{4}, 8.82 \mathrm{~g}$ sodium citrate, $10 \mathrm{mg}$ disodium-EDTA, 1.01 diethylamine $\mathrm{HCl}, 200 \mathrm{mg}$ 1-decanesulfonic acid or SOS), $\mathrm{pH}$ to $6.4 ; 40-70 \mathrm{~mL} \mathrm{CH}_{3} \mathrm{CN} ; 5-10 \mathrm{~mL}$ THF. An amperometric detector, integral to the HPLC, detects electrochemically active compounds in the dialysate and their concentration can be estimated from the peak height recorded on the chart recorder. As stated previously, the first three data points that differ by less than $10 \%$ are used to estimate basal concentrations, and subsequent measurements made following a challenge are converted to a percentage of these values. Ringer's solution was perfused through dialysis probes resting in standards of known concentrations. Peak heights were determined at four different concentrations. Using the average of three peaks at all four concentrations, a line of linear regression was determined. After conversion to the same voltage and amperage used for the standards, peak heights of dialysate were plotted on this line of regression.

\section{Locomotor Activity}

On test day, the animals were placed individually into one of four behavioral boxes (San Diego Instuments, San Diego, CA) for one hour to acclimate to the box. The boxes were $41.3 \times 41.3 \times 30.5 \mathrm{~cm}$ clear acrylic with an electronic system (Photobeam Activity System, San Diego Instruments, San Diego, CA) consisting of 16 infrared beams projecting from side to side and 16 beams from front to back to monitor locomotor activity. Locomotor activity was then recorded in 10-min intervals for a minimum of four hours onto a PC-AT computer using the hardware for the Photobeam Activity System. Data represents the average total locomotor activity over the 100 min time interval following MP administration.

\section{Data Analysis}

Peak increases in extracellular NE and DA are expressed using the 'percentage of basal' values, as indicated above. Post hoc significance testing of univariate analyses employed a Student Newman-Keuls $t$-test.

\section{RESULTS}

\section{Basal Levels}

Basal levels of mPFC NE and DA were calculated as $5.01 \pm 0.21 \mathrm{pg} / 10 \mu \mathrm{L}$ and $4.27 \pm 0.17 \mathrm{pg} / 10 \mu \mathrm{L}$, respectively. For ease of comparison, all further results are expressed as percentage increase over basal.

\section{Brief Handling-induced Stress Response}

Restraint for 15 min produced a 55\% $(p<.001)$ (Figure 1 and Figure 2) increase over baseline in extracellular
DA $(\mathrm{n}=8)$, whereas NE levels were increased $66 \%$ from baseline ( $\mathrm{n}=5, p<.05$, Figure 3 ). Sixty minutes following termination of stress, we observed a trend in DA indicating a 10\% decrease below baseline levels, although this was not statistically significant.

\section{Brief Stress Alteration of DA and NE during MP Challenge}

A robust response of $273 \%$ in DA overflow occurred when animals were handled $15 \mathrm{~min}$ after IG MP (10 $\mathrm{mg} / \mathrm{kg})$ challenge $(\mathrm{n}=6)$. This was significantly higher $(p<.02)$ than both the MP-induced peak DA response of $129 \%$ in stress naïve animals $(n=5)$ (Figure 1$)$ and the peak response seen when animals were only stressed (55\% increase, $p<.003$ ). However, the NE response to MP did not show a significant difference between animals that were handled ( $50 \%$ increase, $\mathrm{n}=4)$ and those that were not $(30 \%$ increase, data not shown, $n=4)$.

\section{Brief Handling-induced Stress $2 \mathrm{~h}$ before MP Challenge Reduces DA But Not NE Overflow}

Animals were challenged with IP MP $(5 \mathrm{mg} / \mathrm{kg})$ two hours following restraint $(n=5)$, which produced a $203 \%$ increase in extracellular DA levels. This was significantly lower $(p<.001)$ (Figure 2$)$ than that observed in stress naïve animals $(286 \%, \mathrm{n}=5)$. There were no significant differences in the changes in NE levels produced by MP challenge between stress-naïve $(127 \%$, $\mathrm{n}=6)$ and stress-exposed animals $(90 \%, \mathrm{n}=4)$ (Figure $3)$, although this data suggests a trend toward a reduced response. As a control, animals were also injected with saline two hours following restraint. This produced a $60 \%$ increase in DA levels $(n=4$, Figure 2$)$, which was identical to the $61 \%$ DA increase following acute saline injection $(n=5)$.

\section{Brief Stress Does Not Alter Locomotor Responses to MP Challenge}

The average of $100 \mathrm{~min}$ of beam crossings following an IP administration of MP is 1200 laser breaks $(n=8)$. This does not differ significantly from the average of 1100 breaks recorded when IP MP is administered $2 \mathrm{~h}$ after a brief 15-min handling stress ( $\mathrm{n}=8$, Figure 4). When the average of $100 \mathrm{~min}$ of beam crossings were recorded following an IG administration of MP, 1300 laser breaks were recorded $(n=8)$. This was not significantly different from the 1660 breaks recorded when handling occurred 15 after the MP injection ( $\mathrm{n}=8$, Figure 4). When the results from the IG administration of MP were compared, the time points of $40 \mathrm{~min}$ to 140 min were used, since the beams could not differentiate between the investigator's hand and animal movements during the 15-min handling stress. 

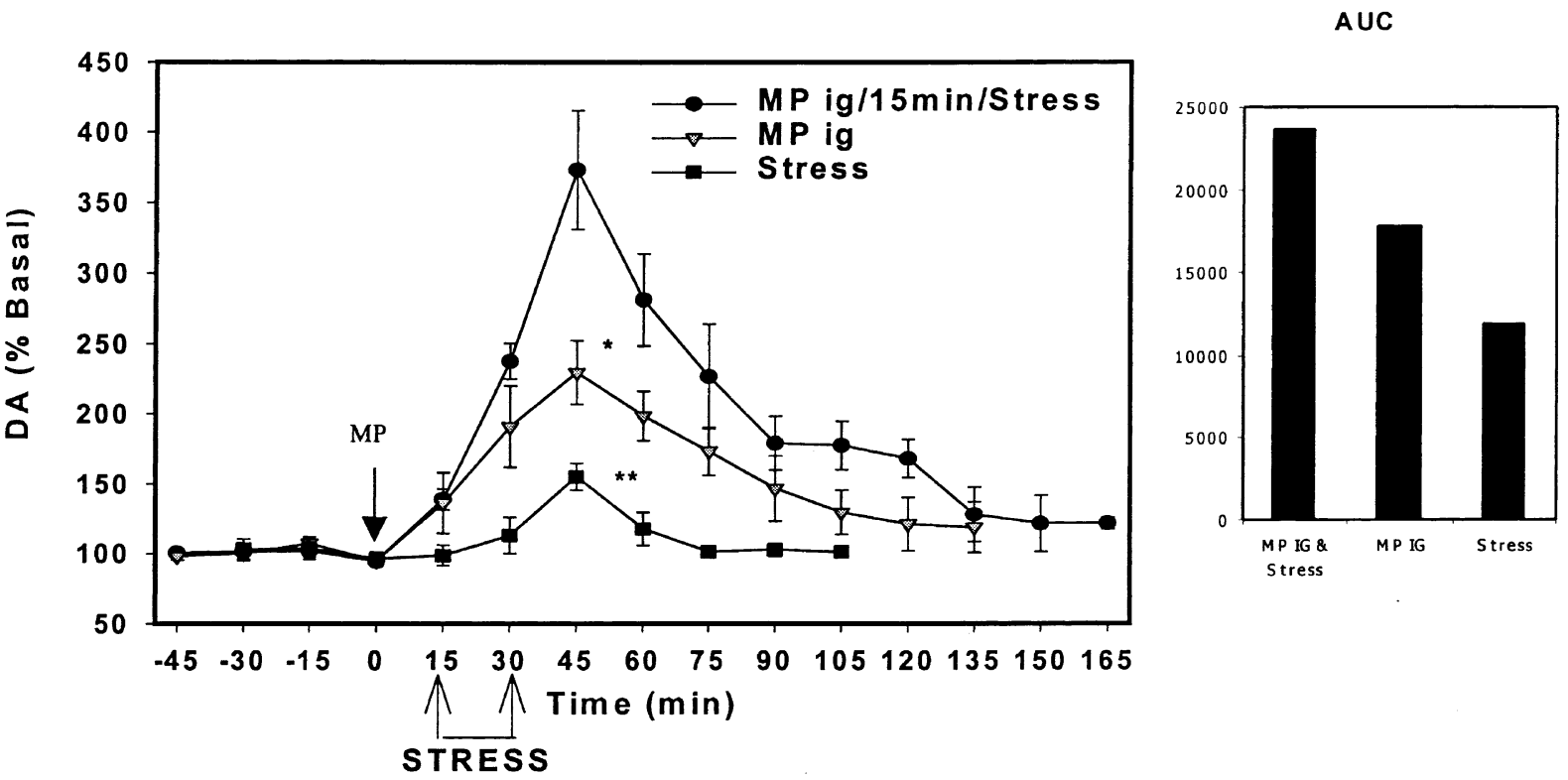

Figure 1. The DA response to handling alone, or to IG MP (10 mg/kg) administered acutely or during stress. Values are expressed as percent of baseline and are mean \pm S.E. $(n=6-8 \text { animals })^{*} p<.02,{ }^{* *} p<.003$ compared with peak effects of the $\mathrm{MP}$-induced increases during stress. Histograms represent the AUC for 0-100 min.

\section{DISCUSSION}

Catecholaminergic systems play an important functional and regulatory role in the pathophysiology of several neuropsychological phenomena including stress and response to the behavioral and therapeutic effects of stimulant drugs. This study demonstrates the modulatory effects that acute handling stress has on MP-induced DA overflow. Consistent with previous findings (Kawahara et al. 2000; Nakane et al. 1994), these data demonstrate that acute handling stress increases DA and NE overflow in the $\mathrm{mPFC}$. The DA responsivity to MP was reduced by $30 \%$ in animals handled $2 \mathrm{~h}$ prior to drug challenge. However, animals given saline (IP) $2 \mathrm{~h}$ after stress showed no difference in DA responsivity from stress naïve animals administered saline (IP). The NE response was also reduced; however, this trend was not statistically significant. Conversely, simultaneous handling potentiated the MP-induced DA response by $212 \%$, while the NE response was not affected. These results suggest that despite similar pharmacokinetic actions of MP on DA and NE transporters, only the effects of MP on the DA system are susceptible to modulation by stress.

It should be noted here that there is a significant difference between dopaminergic and noradrenergic responses to IG MP administration compared with IP MP in stress-naive animals (129\% increase vs. $286 \%$ for DA and $30 \%$ vs. $127 \%$ for NE, see Results section). This trend is in agreement with the results from our previous re- port of the increases in the NAcc DA following IG and IP administration of MP (Gerasimov et al. 2000).

\section{Brief Stress Enhances the DA Response to MP When Administered Simultaneously with IG MP}

MP is a mainstream treatment for attention deficit hyperactivity disorder (ADHD), although the mechanisms of action have not been fully elucidated. We previously demonstrated that MP administered orally in therapeutically relevant doses produces significant increases in extracellular nucleus accumbens DA in rodents (Gerasimov et al. 2000) and striatal DA in the human brain (Volkow et al. 2001). We extended these findings to include a functionally and anatomically related region of the MPFC using IG and IP administration of MP in rats. The stress-induced potentiation of DA responsivity to MP suggests an amplification of mPFC inputs. mPFC neurons are characteristically different from those in the limbic areas, possibly rendering them more responsive to stressful stimuli. In particular, the $\mathrm{mPFC}$ regions which contain ascending midbrain neurons are characterized by a significantly reduced density of presynaptic DA autoreceptors (Horger and Roth 1996). This physiological trait might contribute to an increased rate of VTA DA cell burst firing, which is also correlated to increases in DA overflow in terminal regions like the mPFC (Suaud-Chagny et al. 1992). Since the application of stress was timed to coincide with peak levels of MP- 

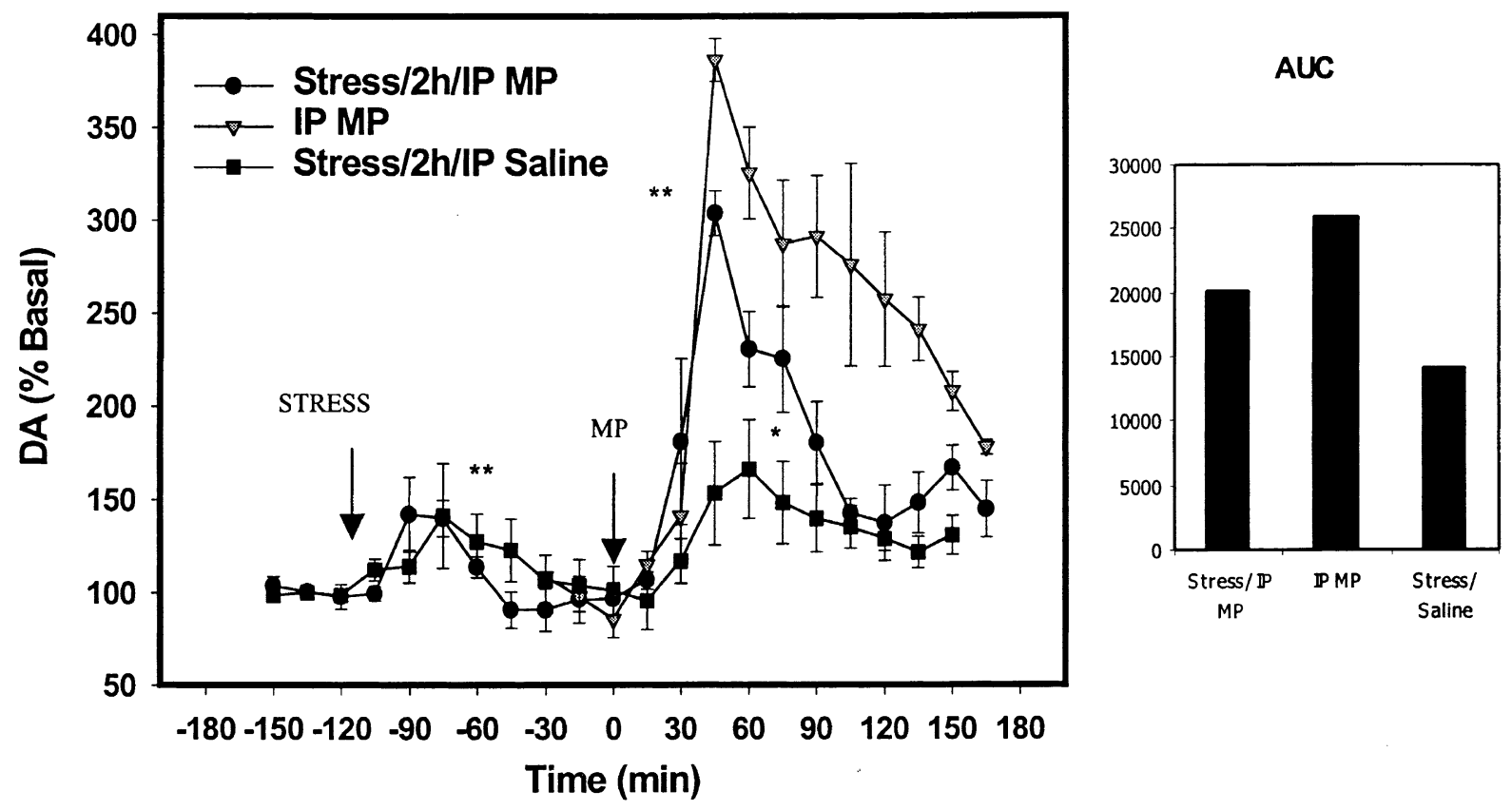

Figure 2. The DA response of saline injection $2 \mathrm{~h}$ after handling stress, or to IP MP $(5 \mathrm{mg} / \mathrm{kg})$ administered acutely or $2 \mathrm{~h}$ after stress. Values are expressed as percent of baseline and are mean \pm S.E. ( $\mathrm{n}=5-6$ animals) ${ }^{*} p<.007,{ }^{* *} p<.001$ compared with peak effects of MP-induced increases $2 \mathrm{~h}$ after stress. Stress alone is compared with baseline levels, ${ }^{* *} p<.001$. Histograms represent the AUC for 0-100 min.

induced DA overflow, a compensatory decrease in tonic DA neuronal regulation of firing rates might be responsible for the observed stress-induced amplification of DA transmission. The DA response could be additive in nature due to individual challenges of stress or MP acting independently of one another.

Gamma-amino butyric acid (GABA)-ergic and glutamatergic systems might also contribute to this effect. Enrico et al. (1998) demonstrated that infusion of a $\mathrm{GABA}_{\mathrm{B}}$ agonist (baclofen), though not a $\mathrm{GABA}_{\mathrm{A}}$ agonist (muscimol) suppressed stress-induced increases of extracellular mPFC DA. These findings are of particular interest in light of the clinical anxiolytic utility of $\mathrm{GABA}_{\mathrm{A}}$ agonists. This same report indicated that glutamatergic antagonists (CPP, AP-5 and CNQX) also suppressed stress-induced increases presumably through reducing tonic activation of DA by antagonizing excitatory glutamatergic input. It should be noted that in these studies a high concentration of calcium in the perfusion solution was used, which is known to result in increased neuronal release of DA. Additionally, findings by Doherty and Gratton (1999) indicate that infusion of a $\mathrm{GABA}_{\mathrm{A}}$ agonist (muscimol) directly into the PFC inhibits stress-induced release of DA in the $\mathrm{MPFC}$, while a $\mathrm{GABA}_{B}$ antagonist (phaclofen) enhances this same release. On the contrary, under similar conditions Feenstra et al. (Feenstra et al. 1998) found no influence of the locally applied $\mathrm{GABA}_{\mathrm{B}}$ agonist baclofen or glutamate receptor agonists and antagonists (ACPD, dixocilpine) in the mPFC, although in an earlier study (Feenstra et al. 1995) this group reported a benzodiazepine (diazepam) to inhibit the stress-induced release of DA in the mPFC. Taken together, these studies indicate that $\mathrm{GABA}_{\mathrm{B}}$ antagonism and $\mathrm{GABA}_{\mathrm{A}}$ agonism could play a possible role in the enhanced DAergic response to MP challenge in the mPFC induced by stress, although additional investigations would be required to support this hypothesis.

\section{Brief Stress Attenuates the DA Response When Administered $2 h$ prior to IP MP}

DA burst firing in the mPFC is dependent on afferent input from rostral brain regions (Grace 2000). The observed 30\% attenuation in DA overflow indicates that during the 2-h interlude between stress and MP administration, an activation of autoreceptors might have altered tonic DA neuronal firing, thereby contributing to the reduced response to MP following stress. Results of our own control study indicate the DA response to IP saline following stress is not statistically different from this response in stress naïve animals. Thus, one could suggest that the MPFC stress-induced inhibition of DAergic activity is not mediated through a reduction of endogenous DA reserves, but rather through indirect pathways, such as the GABAergic or glutamatergic modulation.

Another possible mediator of the DAergic response is blockade of the NET by MP. This is consistent with the demonstrated affinity of MP for NET (Gatley et al. 


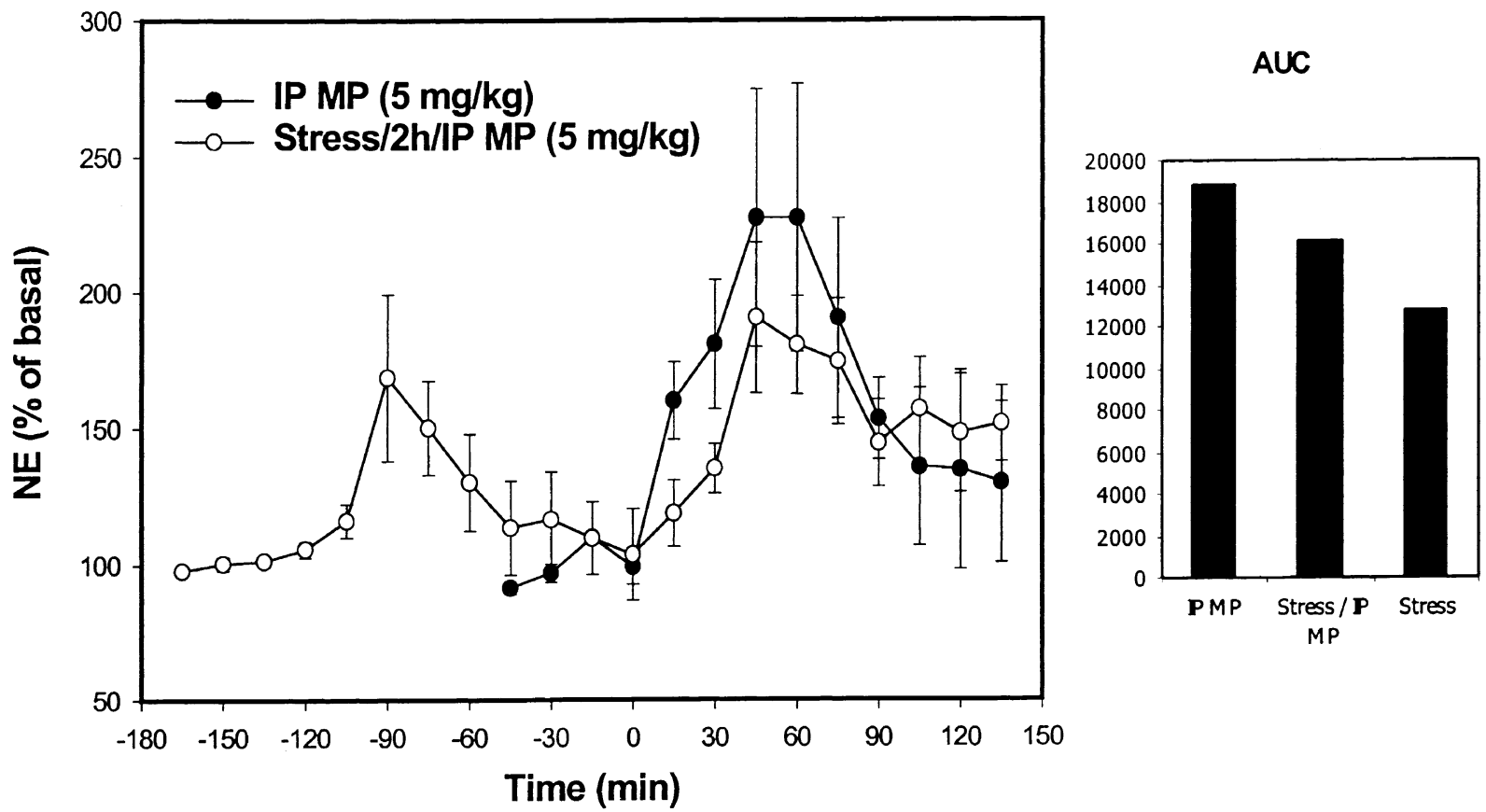

Figure 3. The NE response to IP MP ( $5 \mathrm{mg} / \mathrm{kg})$ administered acutely or $2 \mathrm{~h}$ after stress. There was a trend for a reduction in the response after stress, although this was not significant. Stress alone is compared with baseline levels, ${ }^{*} p<.05$. Histograms represent the AUC for 0-100 min.

1996), and the report that the NET has a higher affinity for DA than for NE (Raiteri et al. 1977). The influential role of the NET in regulating DA overflow in the $\mathrm{MPFC}$ is further supported by a study resulting in significant DA increase after infusion of selective NET blocker desipramine (Gresch et al. 1995). Interestingly, several studies suggest that changes in DA release during and following stress are not affected by andrenolectomy
(Imperato et al. 1991), or by 6-OH-DA lesions of the LC (Kawahara et al. 1999).

\section{Noradrenergic Response to MP Challenge and Stress}

According to our results, NEergic response to MP does not appear to be affected by stress. This suggests that the NE system in the $\mathrm{mPFC}$ is regulated in a dissimilar

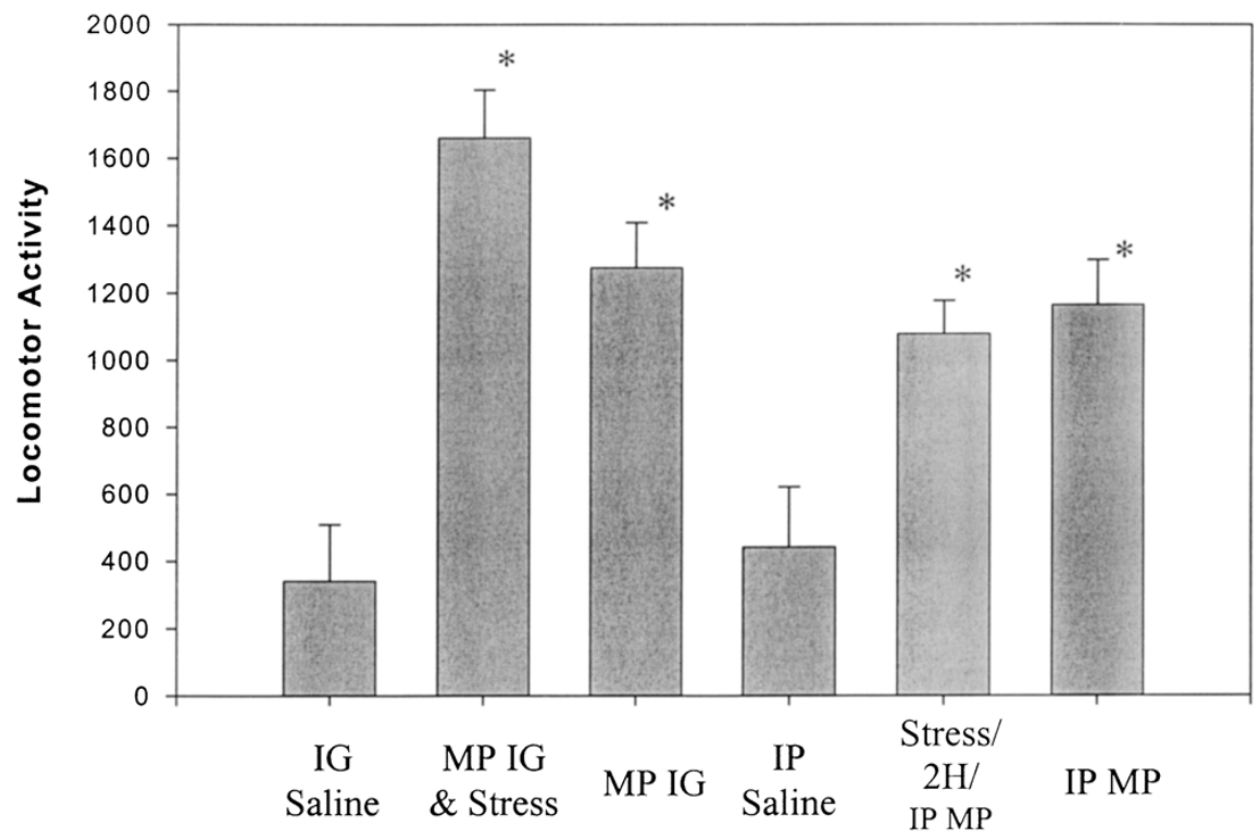

Figure 4. The average of 100 min of beam crossings following an IP or IG administration of MP or saline acutely, following $2 \mathrm{~h}$ handling, or during simultaneous handling $(\mathrm{n}=8$, each group). Administration of MP is significantly greater than saline injection $\left({ }^{*} p<.05\right)$, but there is not significance between MP groups. 
manner from the DA system, which might be a function of inputs from several limbic brain regions. For example, infusion of $\mathrm{GABA}_{\mathrm{A}}$ and $\mathrm{GABA}_{\mathrm{B}}$ antagonists (bicuculline and CGP 52432, respectively) into the LC did not alter the handling-induced increases of $\mathrm{NE}$ in the $\mathrm{MPFC}$ (Kawahara et al. 2000). In the same study, a non-NMDA glutamate receptor antagonist (DNQX) did not alter stressinduced changes in the mPFC NE overflow, whereas infusion of an NMDA glutamate receptor antagonist (CPP) reduced the stress-induced response of $\mathrm{MPFC}$ NE. Additionally, direct infusion of the $\mathrm{GABA}_{\mathrm{B}}$ agonist baclofen or glutamate receptor agonist and antagonists (ACPD, dixocilpine) into the mPFC did not alter stress-induced NE increases in this same region (Feenstra et al. 1998).

\section{MP-induced Locomotor Activity is Not Affected by Acute Stress}

Our results suggest that acute stress does not alter MPinduced locomotor activity. On the other hand, chronic stress decreases open field locomotor activity (Carrizo et al. 1997), therefore it would be of relevance to investigate the effect of chronic stress on acute MP response. In addition, studies involving MP administered chronically followed by acute stress would aid in the elucidation of neurobehavioral processes underlying ADHD.

In conclusion, stress distinctly modulates the response of the DAergic system to a therapeutically relevant dose of MP, whereas the NE response appears to be spared. In agreement with a partial involvement of NE in the locomotor response to psychostimulants (Svensson and Ahlenius 1983), our results also suggest that locomotor activity is not a predictable measure of the interaction between stress and MP. To this end, increases in DAergic transmission in the mesocorticolimbic system appear to be strongly context dependent. These findings could contribute to our understanding of the complex mechanisms underlying the therapeutic action of MP. Additional studies exploring the involvement of other brain areas in the interaction between environmental stimuli and catecholaminergic response to MP are currently underway.

\section{ACKNOWLEDGMENTS}

This research was carried out at Brookhaven National Laboratory under contract with the U.S. Department of Energy Office of Biological and Environmental Research (USDOE/OBER DE-AC02-98CH10886), and by the National Institutes of Mental Health (NIMH MH49165 and NIMH R2955155) and the National Institute on Drug Abuse (5RO-DA06278 and DA09490).

\section{REFERENCES}

Abercrombie ED, Keefe KA, DiFrischia DS, Zigmond MJ (1989): Differential effect of stress on in vivo dopamine release in striatum, nucleus accumbens, and medial frontal cortex. J Neurochem 52:1655-1658

Carrizo E, Cano G, Suarez-Roca H, Bonilla E (1997): Motor activity and quantitative autoradiographic analysis of muscarinic receptors in the brain of rats subjected to the forced swimming test. Brain Res Bull 42:133-139

Doherty MD, Gratton A (1999): Effects of medial prefrontal cortical injections of GABA receptor agonists and antagonists on the local and nucleus accumbens dopamine responses to stress. Synapse 32:288-300

Enrico P, Bouma M, de Vries JB, Westerink BH (1998): The role of afferents to the ventral tegmental area in the handling stress-induced increase in the release of dopamine in the medial prefrontal cortex: a dual-probe microdialysis study in the rat brain. Brain Res 779:205-213

Feenstra MG, Botterblom MH, van Uum JF (1995): Noveltyinduced increase in dopamine release in the rat prefrontal cortex in vivo: inhibition by diazepam. Neurosci Lett 189:81-84

Feenstra MG, Botterblom MH, van Uum JF (1998): Local activation of metabotropic glutamate receptors inhibits the handling-induced increased release of dopamine in the nucleus accumbens but not that of dopamine or noradrenaline in the prefrontal cortex: comparison with inhibition of ionotropic receptors. J Neurochem 70: 1104-1113

Feenstra MG, Teske G, Botterblom MH, De Bruin JP (1999): Dopamine and noradrenaline release in the prefrontal cortex of rats during classical aversive and appetitive conditioning to a contextual stimulus: interference by novelty effects. Neurosci Lett 272:179-182

Gatley SJ, Pan D, Chen R, Chaturvedi G, Ding YS (1996): Affinities of methylphenidate derivatives for dopamine, norepinephrine and serotonin transporters. Life Sci 58:231-239

Gerasimov MR, Franceschi M, Volkow ND, Gifford A, Gatley SJ, Marsteller D, Molina PE, Dewey SL (2000): Comparison between intraperitoneal and oral methylphenidate administration: A microdialysis and locomotor activity study. J Pharmacol Exp Ther 295:51-57

Grace AA (2000): The tonic/phasic model of dopamine system regulation and its implications for understanding alcohol and psychostimulant craving. Addiction 95(Suppl 2):S119-128

Gresch PJ, Sved AF, Zigmond MJ, Finlay JM (1995): Local influence of endogenous norepinephrine on extracellular dopamine in rat medial prefrontal cortex. J Neurochem 65:111-116

Horger BA, Roth RH (1996): The role of mesoprefrontal dopamine neurons in stress. Crit Rev Neurobiol 10:395-418

Ihalainen JA, Riekkinen P Jr, Feenstra MG (1999): Comparison of dopamine and noradrenaline release in mouse prefrontal cortex, striatum and hippocampus using microdialysis. Neurosci Lett 277:71-74

Imperato A, Puglisi-Allegra S, Casolini P, Angelucci L (1991): Changes in brain dopamine and acetylcholine release during and following stress are independent of the pituitary-adrenocortical axis. Brain Res 538:111-117

Kawahara H, Kawahara Y, Westerink BH (2000): The role of afferents to the locus coeruleus in the handling stressinduced increase in the release of noradrenaline in the medial prefrontal cortex: a dual-probe microdialysis study in the rat brain. Eur J Pharmacol 387:279-286 
Kawahara Y, Kawahara H, Westerink BH (1999): Comparison of effects of hypotension and handling stress on the release of noradrenaline and dopamine in the locus coeruleus and medial prefrontal cortex of the rat. Naunyn Schmiedebergs Arch Pharmacol 360:42-49

Kuczenski R, Segal DS (1997): Effects of methylphenidate on extracellular dopamine, serotonin, and norepinephrine: comparison with amphetamine. J Neurochem 68:2032-2037

Kuczenski R, Segal DS (2001): Locomotor effects of acute and repeated threshold doses of amphetamine and methylphenidate: relative roles of dopamine and norepinephrine. J Pharmacol Exp Ther 296:876-883

Mathe JM, Nomikos GG, Blakeman KH, Svensson TH (1999): Differential actions of dizocilpine (MK-801) on the mesolimbic and mesocortical dopamine systems: role of neuronal activity. Neuropharmacology 38:121-128

Nakane H, Shimizu N, Hori T (1994): Stress-induced norepinephrine release in the rat prefrontal cortex measured by microdialysis. Am J Physiol 267:R1559-1566

Raiteri M, Del Carmine R, Bertollini A, Levi G (1977): Effect of sympathomimetic amines on the synaptosomal transport of noradrenaline, dopamine and 5-hydroxytryptamine. Eur J Pharmacol 41:133-143
Shimizu N, Nakane H, Hori T, Hayashi Y (1994): CRF receptor antagonist attenuates stress-induced noradrenaline release in the medial prefrontal cortex of rats. Brain Res 654:145-148

Suaud-Chagny MF, Chergui K, Chouvet G, Gonon F (1992): Relationship between dopamine release in the rat nucleus accumbens and the discharge activity of dopaminergic neurons during local in vivo application of amino acids in the ventral tegmental area. Neuroscience 49:63-72

Svensson L, Ahlenius S (1983): Suppression of exploratory locomotor activity by the local application of dopamine or 1-noradrenaline to the nucleus accumbens of the rat. Pharmacol Biochem Behav 19:693-699

Van Gaalen M, Kawahara H, Kawahara Y, Westerink BH (1997): The locus coeruleus noradrenergic system in the rat brain studied by dual-probe microdialysis. Brain Res 763:56-62

Volkow ND, Wang G, Fowler JS, Logan J, Gerasimov M, Maynard L, Ding Y, Gatley SJ, Gifford A, Franceschi D (2001): Therapeutic doses of oral methylphenidate significantly increase extracellular dopamine in the human brain. J Neurosci 21:RC121 